\title{
Effect of coil size on transcranial magnetic stimulation (TMS) focality
}

Hedyeh Bagherzadeh, Fow-sen Choa

Hedyeh Bagherzadeh, Fow-sen Choa, "Effect of coil size on transcranial magnetic stimulation (TMS) focality," Proc. SPIE 11020, Smart Biomedical and Physiological Sensor Technology XV, $110200 Z$ (2 May 2019); doi: $10.1117 / 12.2524503$

Event: SPIE Defense + Commercial Sensing, 2019, Baltimore, Maryland, United States 


\title{
Effect of coil size on transcranial magnetic stimulation (TMS) focality
}

\author{
Hedyeh Bagherzadeh ${ }^{1}$, Fow-sen Choa* ${ }^{1}$ \\ ${ }^{1}$ University of Maryland Baltimore County, Department of Computer Science and Electrical \\ Engineering, MD, USA
}

\begin{abstract}
In recent years, there is an increasing interest in noninvasive treatments for neurological disorders like Alzheimer and Depression. Transcranial magnetic stimulation (TMS) is one of the most effective methods used for this purpose. The performance of TMS highly depends on the coils used for the generation of magnetic field and induced electric field particularly their designs affecting depth and focality tradeoff characteristics. Among a variety of proposed and used TMS coil designs, circular coils are commonly used both in research and medical and clinical applications. In current study, we focus on changing the outer and inner sizes (diameter) and winding turns of ring coils and try to reach deeper brain regions without significant field strength decay. The induced electric field and the decay rate of the generated field with depth were studied with finite element method calculations. The results of the performed simulations indicate that larger diameter coils have a larger equivalent field emission aperture and produce larger footprint of induced electric field initially. However, their emission solid angles are smaller and, as a result, the field divergence or the decay rates of the generated field with depth are smaller as well, which give them a good potential to perform better for deep brain stimulation compared with that of smaller coil.
\end{abstract}

Keywords: TMS, coil, electric field, magnetic field, decay rate, focality

\section{INTRODUCTION}

Transcranial Magnetic Stimulation (TMS) is a non-invasive and painless neurostimulation and neuromodulation method which has been widely used for treatment of neurological and psychiatric disorders like Alzheimer and Depression. The basis of TMS is inducing electric field through the scalp via a magnetic field generated by a wire coil. This induced electric field excites the neurons in a specific area of the brain; for example exciting motor cortex can result in a twitch in the targeted muscle. TMS is a rapidly developing technique for exciting brain and helping patients with neurological disorders. Researchers around the world study different aspects of TMS to improve the performance and efficiency of the apparatus to help patients.

\subsection{TMS basics}

The effectiveness of TMS has been approved by a variety of experiments starting from mid-twentieth century using animal studies. These studies showed the first evidence of the effect of an electric pulse applied to the motor cortex of the animals. In these studies, the brain was exposed by removing the skull for a better analysis of animal's behavior under the applied electric field [1]. These types of experiments are still performed to study the efficiency of TMS apparatus. About 4 decades ago, in 1980, Merton and Morton used human subject for their electrical brain stimulation and for the first time, showed that Transcranial Electric Stimulation (TES) can be used to electrically stimulate human brain without removing scalp. Their high-voltage electric shock excited the neurons in motor cortex and resulted in a motor evoked potential and led to a twitch in the subject's arm muscles. But unfortunately, their tests resulted in an induced local pain in the location of the electrodes [2]. In 1985, substituting TES with TMS was proposed by Barker. Et al. They studied the induction of electric field through generation of magnetic field using a wire-wrapped coil [1]. If the wire coil is located parallel to the subject's scalp, the generated magnetic field is in a perpendicular plane and induced electric field is in a parallel plane to both the coil and the scalp. Although the intensity of the induced electric field reduces because of extra-cerebral tissues, it is still adequate to excite and depolarize neurons and generate activity in the neural network [3].

\footnotetext{
*choa@umbc.edu; phone: (410) 455-3272; https://blogs.cs.umbc.edu/choalab/
} 


\subsection{TMS apparatus}

As mentioned before, a wire-wrapped coil is used to generate the required magnetic field for the induction of electric field. The performance of TMS depends on these coils and their design since the design can affect the focality and intensity of the generated and the induced field that penetrates the skull and excites the neurons. A variety of coil designs have been proposed and used: circular coil which is the first and most conventional type of coil used for TMS, Figure 8 coil proposed by Uneo et al. (1988) for the purpose of localizing brain stimulation resulting in higher current densities in targeted areas [4], H-coil designed by Roth et al. (2002) to stimulate deep brain regions [5], halo coil proposed by ishii et al. (2008) for deep TMS (dTMS) [6], double cone or tilted double cone and other designs. The mentioned designs are shown in figure 1.

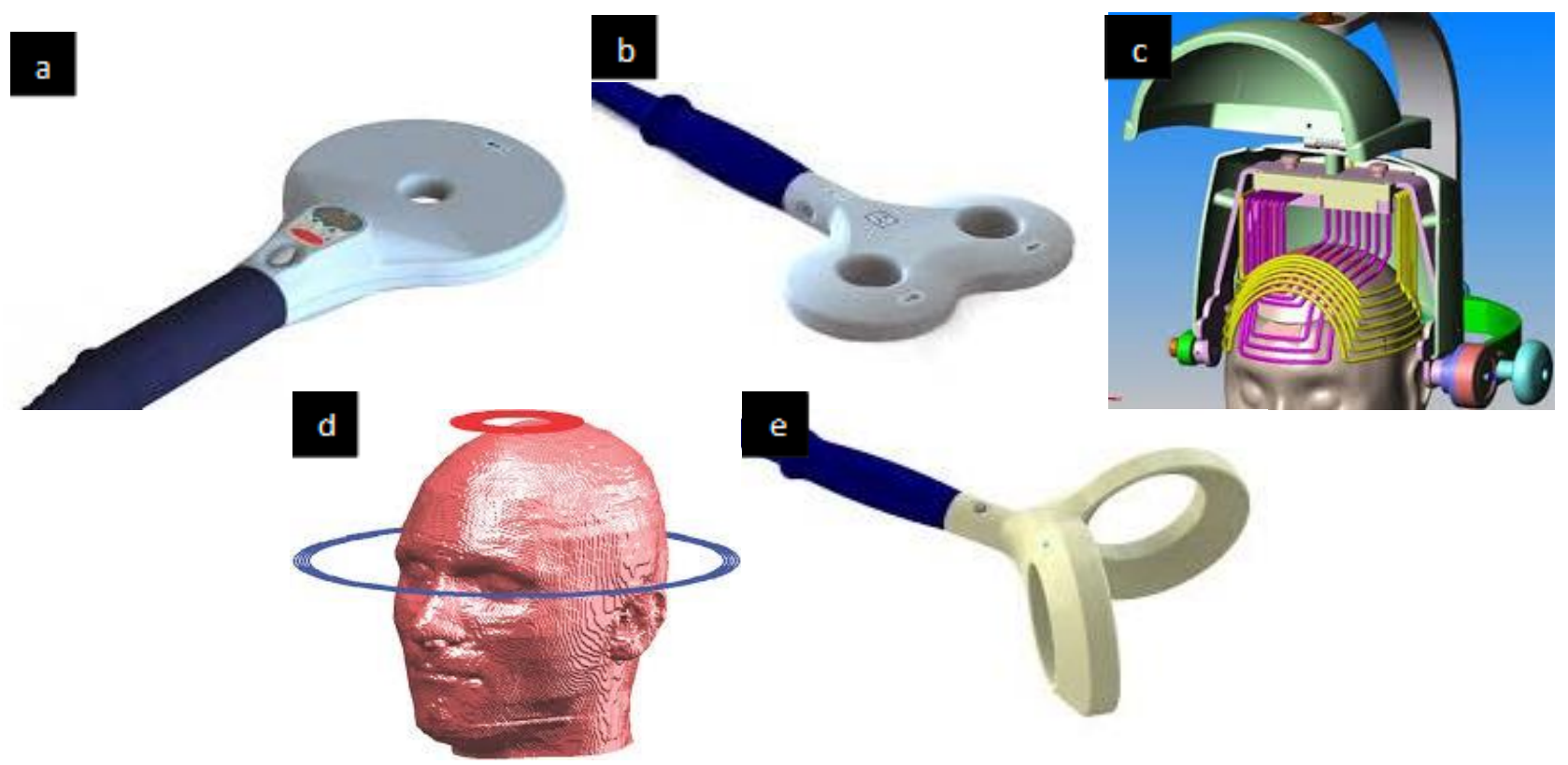

Figure 1: Conventional TMS coils: a) Magstim circular coil, b) Magstim figure 8 coil, c) H-coil, d) Halo coil, e)

Magstim double cone coil

As seen above, these coils are designed for a specific purpose and their performance is relatively different. Researchers have been performing experiments and simulations on these coils to study their behavior and the efficiency of the field generated by them for stimulation of the targeted areas in the brain. In a study by Roth et al. (2007), the performance of figure 8 coil and deep $\mathrm{H}$-coil was investigated using finite element simulations; they concluded that although $\mathrm{H}$-coils have the ability to effectively stimulate deeper regions of the brain, they have a wider electric field distribution which is not satisfactory [7]. Deng et al. (2012) studied 50 different coils and their electric field distributions using finite element simulations based on a spherical human head model. They concluded that there is a trade-off between focality and intensity of the induced electric field meaning that deep brain stimulation is accompanied with a spread of generated field resulting in less focality [8]. In another study Lu \& Ueno (2017), compared the induced field from conventional TMS coils. Their simulations suggested that higher electric fields in deeper subregions of the brain are generated by double cone or halo coils. They also had the same conclusion as Deng et al. (2012): a trade-off between focality and intensity for deep brain stimulation [9]. Meng et al. (2017) proposed a coil design to generate maximized electromagnetic field gradient. The proposed design included two coils, running current along opposite directions resulting in anti-phase operations which leads to higher electric field gradients with lower current [10].

\subsection{Current study}

It was noted previously that focality and intensity of the induced electric field in TMS, have a trade-off for deep brain stimulation; thus, understanding the decay rate of the electric field for each coil in the brain is of high importance. In this work, circular coils, as the most conventional type of coils, with different sizes and diameters are considered and their generated field, in accordance with different depths, is studied and analyzed. In the following sections of this paper, methodology, results and conclusions will be discussed. 


\section{METHODOLOGY}

In order to perform the analysis for this study, the coils' structures were built in COMSOL Multiphysics (Version 5.3, http://www.comsol.com). The inner radius of the coils represents the radius of the inner core, which in this paper is introduced as silicon steel while the outer radius of the coils represents the total radius of the simulated coils. The conductivity of the silicon steel in the current study is $0[\mathrm{~S} / \mathrm{m}]$ and the relative magnetic permeability $(\mu)$ is set to 4 in COMSOL Multiphysics software; the coil material is also considered to be Copper. To study the effect of the inner and outer radius of the coil on the induced electric field in different distance from the surface of a flat coil, three sets of simulations with these conditions were performed. In order to perform these simulations in COMSOL Multiphysics, a frequency of $1 \mathrm{MHz}$ is used.

In the first set of simulations, the inner radius of the coils is kept constant at $7.5 \mathrm{~cm}$ and the outer radius is considered to be from $12 \mathrm{~cm}$ to $28 \mathrm{~cm}$, in $4 \mathrm{~cm}$ increments, as depicted in figure 2-a; While for the second set of the performed simulations, the outer radius of the coil is fixed at $24 \mathrm{~cm}$ and the inner radius varied from $3.5 \mathrm{~cm}$ to $15.5 \mathrm{~cm}$ in an increment of $4 \mathrm{~cm}$ (shown in Figure 2-b). For the third set of the simulations, both inner and outer radii of the studied coils were changed. In the latest set of simulations, for each coil the values of $0.5 \mathrm{~cm}$ and $3 \mathrm{~cm}, 1.5 \mathrm{~cm}$ and $6 \mathrm{~cm}, 3.5 \mathrm{~cm}$ and $12 \mathrm{~cm}$ and $7.5 \mathrm{~cm}$ and $24 \mathrm{~cm}$ have been considered for inner and outer radius, respectively (Figure 2-c). For all three sets of simulations, the length of the coils is kept constant at $12 \mathrm{~cm}$.

Since the reported data is normalized to the maximum induced electric field which is observed in a location very close to the coil, the number of the turns as well as the current passing through each turn of the coils is not mentioned and analyzed. The electric current in the coil driver circuit, is tuned to prevent magnetic saturation in the core material. The environment (model) is a sphere filled with air. The conductivity of the target area is zero, because in the real human head model the conductivity in different regions of the human head is too small to cause any magnetic field distortion which is a phenomenon that might happen in superconductors. The plane parallel to the surface of the coil in the air environment has been selected as the target area to measure the maximum induced electric field in these simulations. This distance, from coil surface, changes from $0.5 \mathrm{~cm}$ to $25 \mathrm{~cm}$, in step size of $0.5 \mathrm{~cm}$.

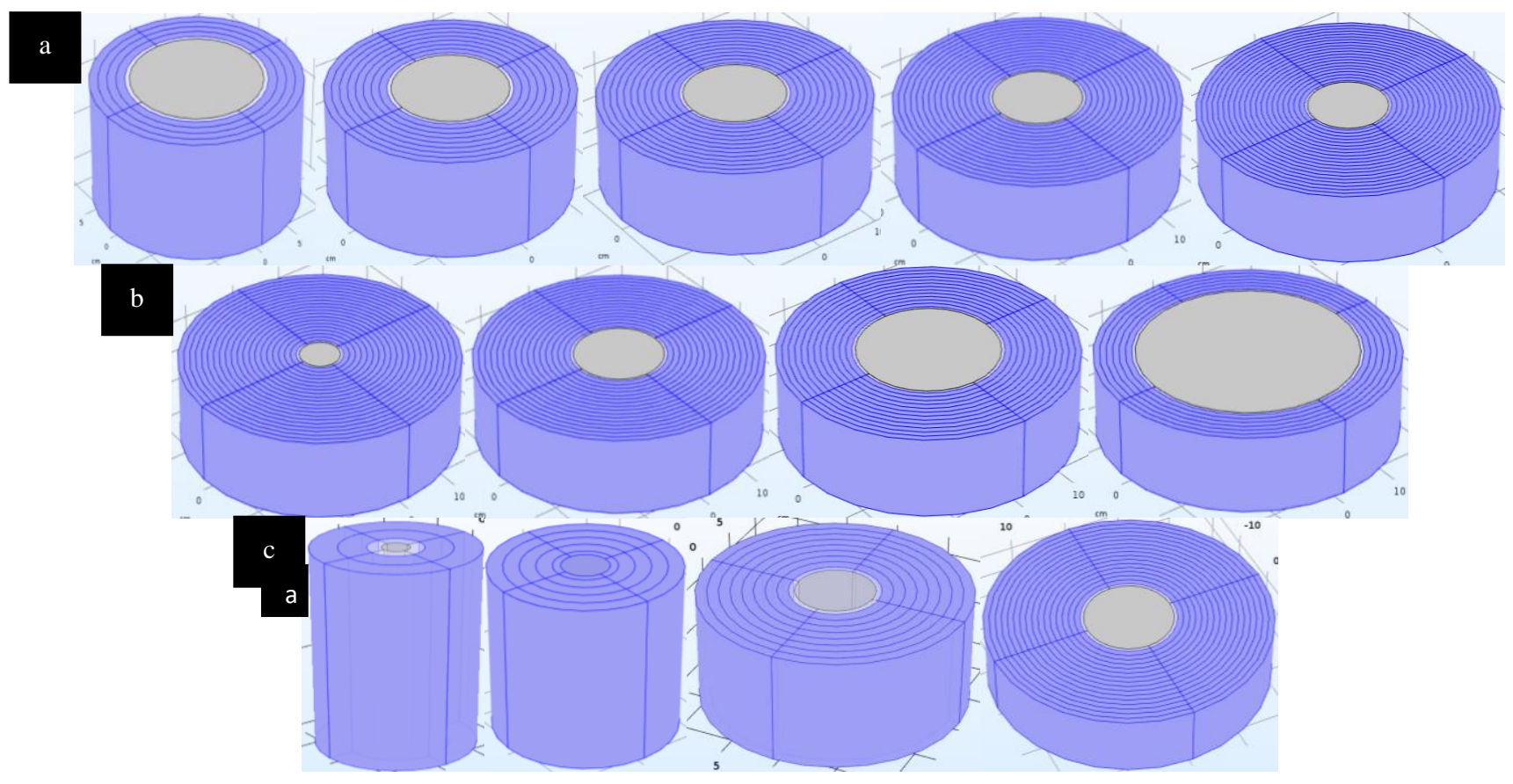

Figure 2: Constructed wire coils in COMSOL, a) constant inner radius of 7.5 and outer radius varying from $12 \mathrm{~cm}$ to $28 \mathrm{~cm}$ in $4 \mathrm{~cm}$ increments from left to right, b) constant outer radius of $24 \mathrm{~cm}$ with varying inner radius from $3.5 \mathrm{~cm}$ to $15.5 \mathrm{~cm}$ in $4 \mathrm{~cm}$ increments from left to right, c) varying inner and outer radius with values of $0.5 \mathrm{~cm}$ and $3 \mathrm{~cm}, 1.5 \mathrm{~cm}$ and $6 \mathrm{~cm}, 3.5$ $\mathrm{cm}$ and $12 \mathrm{~cm}$ and $7.5 \mathrm{~cm}$ and $24 \mathrm{~cm}$, respectively from left to right. 


\section{RESULTS AND DISCUSSION}

After constructing all the models and performing the 3 sets of simulations in COMSOL, the following data was obtained. All the data presented herein are normalized to the maximum electric field captured in the vicinity of the coil (very close to the coil). This normalization helps in better analysis and understanding the decay rate of the electric field for each coil as the depth increases. The results of the simulations are as follows:

\subsection{Fixed inner radius}

In this set of simulations, the inner radius of the studied coils is kept constant at $7.5 \mathrm{~cm}$ while the outer radius changes. Figure 3 depicts the normalized data obtained for this set of simulations. It can be observed that increasing the outer radius of the coil with a constant inner (core) radius leads to higher normalized values of induced electric field which is a result of higher magnetic field generation due to a larger coil. By comparing the decay rate of coils, the data shows $79 \%$ decay for outer radius of $12 \mathrm{~cm}, 70 \%$ decay for outer radius of $20 \mathrm{~cm}$ and $65 \%$ decay for outer radius of $28 \mathrm{~cm}$ for $10 \mathrm{~cm}$ distance from the surface of the coil and $94 \%$ decay for outer radius of $12 \mathrm{~cm}, 91 \%$ decay for outer radius of $20 \mathrm{~cm}$ and $90 \%$ decay for outer radius of $28 \mathrm{~cm}$ for $20 \mathrm{~cm}$ from the surface of the coil. This analysis indicates that an increase in the circular coil's radius decreases its decay rate with depth and increases the coil's efficiency for deep brain stimulation. It should be noted that increasing the outer radius from $24 \mathrm{~cm}$ to $28 \mathrm{~cm}$ does not have a significant effect on the induced electric field; also, after the depth of $20 \mathrm{~cm}$, the normalized values of the electric field are very close, showing the insignificant effect of changing the outer radius of the coil on decay rate in these depths.

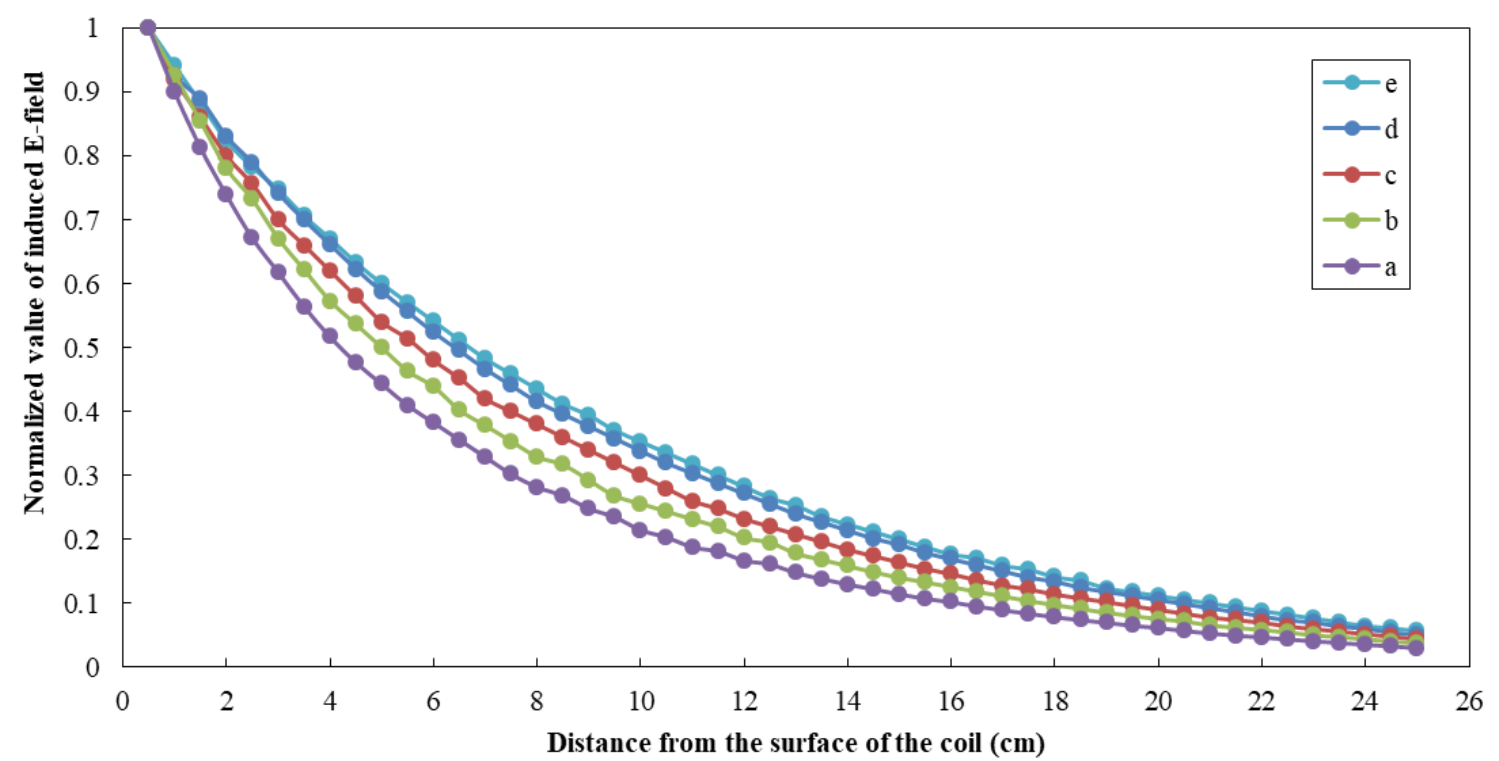

Figure 3: Normalized value of induced electric field for circular flat coils with constant inner radius of $7.5 \mathrm{~cm}$ and outer radius of a) $12 \mathrm{~cm}$, b) $16 \mathrm{~cm}$, c) $20 \mathrm{~cm}$, d) $24 \mathrm{~cm}$, e) $28 \mathrm{~cm}$.

\subsection{Fixed outer radius}

In this dataset, the outer radius of the flat circular coils is fixed at $24 \mathrm{~cm}$ while the inner radius changes from $3.5 \mathrm{~cm}$ to $15.5 \mathrm{~cm}$ in $4 \mathrm{~cm}$ increments. Figure 4 shows the normalized electric field values obtained for this dataset. As shown in figure 4, it can be stated that increasing the inner radius of the coil with a constant outer radius, in other words, increasing the size of the Silicone Steel core, slightly deceases the normalized values of the induced electric field. This incident is caused by a decrease in number of wire turns as the core radius increases. By comparing the decay rate of these coils, the data shows $66 \%$ decay for inner radius of $3.5 \mathrm{~cm}, 66 \%$ decay for inner radius of $7.5 \mathrm{~cm}$ and $69 \%$ decay for inner radius of $15.5 \mathrm{~cm}$ for $10 \mathrm{~cm}$ distance from the surface of the coil and $90 \%$ decay for inner radius of $3.5 \mathrm{~cm}$, $90 \%$ decay for inner radius of $7.5 \mathrm{~cm}$ and $91 \%$ decay for inner radius of $15.5 \mathrm{~cm}$ for $20 \mathrm{~cm}$ distance from the surface of the coil. This analysis indicates that an increase in the circular coil's inner radius with a constant outer radius slightly increases its decay rate with depth and decreases the coil's efficiency for deep brain stimulation. It should be noted that the changes in normalized value of electric field for theses simulations are insignificant; also after the distance of $20 \mathrm{~cm}$ 
from the surface of the coil, the normalized values of the induced electric field are almost similar to each other, showing the insignificant effect of changing the size of the coil on decay rate after $20 \mathrm{~cm}$ distance from the surface of the coil.

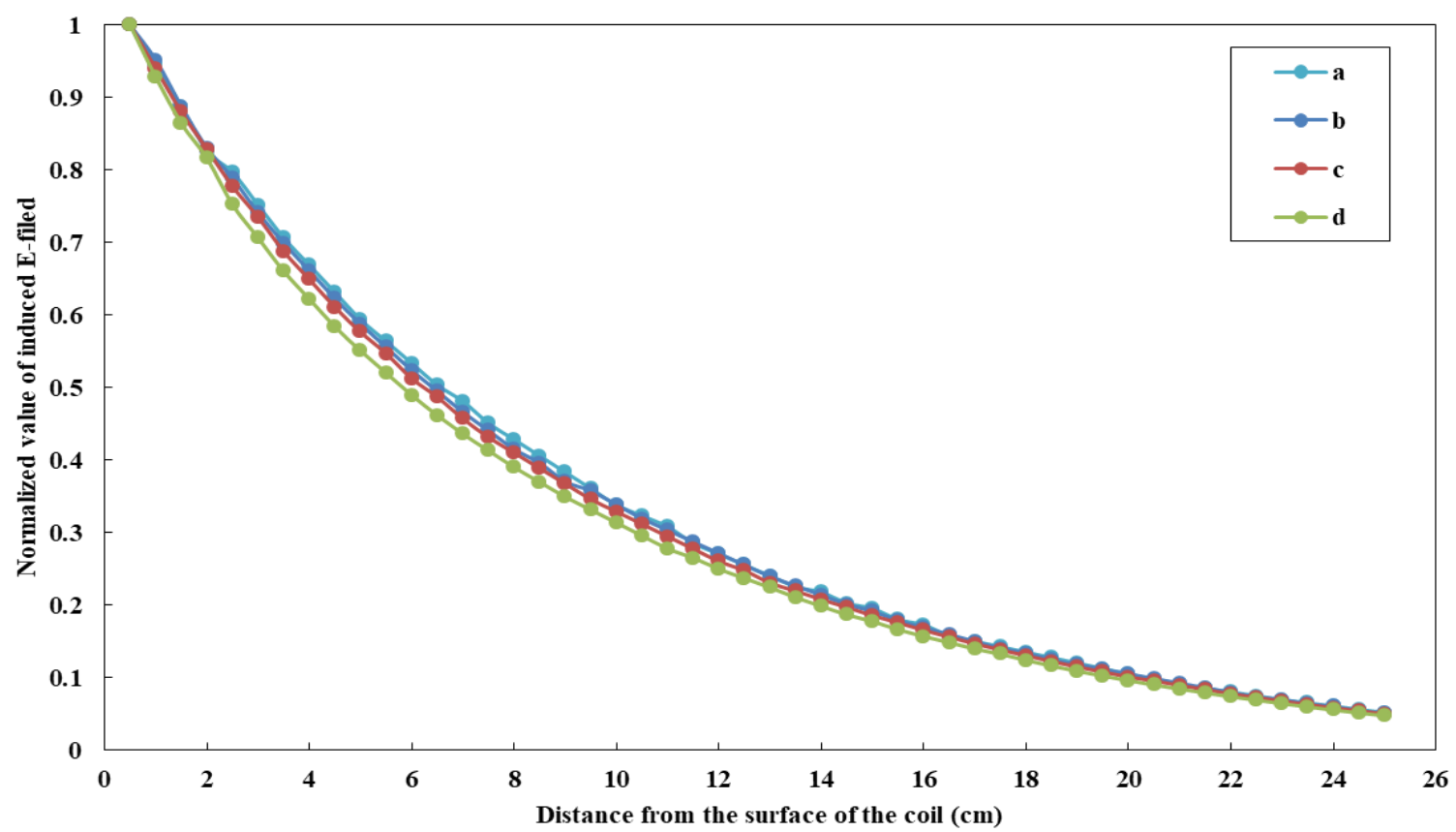

Figure 4: Normalized value of induced electric field for circular flat coils with constant outer radius of $24 \mathrm{~cm}$ and inner radius of a) $3.5 \mathrm{~cm}$, b) $7.5 \mathrm{~cm}$, c) $11.5 \mathrm{~cm}$, d) $15.5 \mathrm{~cm}$

\subsection{Varying inner and outer radii}

In this set of simulations, the inner and outer radius of the flat circular coils is changing and for the 4 datasets are respectively $0.5 \mathrm{~cm}$ and $3 \mathrm{~cm}, 1.5 \mathrm{~cm}$ and $6 \mathrm{~cm}, 3.5 \mathrm{~cm}$ and $12 \mathrm{~cm}$ and $7.5 \mathrm{~cm}$ and $24 \mathrm{~cm}$. Figure 5 is the summary of the data obtained for the normalized induced electric field values from the performed simulations. As shown in figure 5, increasing the size of the coil (both inner and outer radii), increases the normalized values of electric field which agrees with the achieved results from the other datasets. By comparing the decay rate of the coils, the data depicts $92 \%$ decay for inner and outer radii of 0.5 and $3 \mathrm{~cm}, 87.5 \%$ decay for inner and outer radii of 1.5 and $6 \mathrm{~cm}, 79 \%$ decay for inner and outer radii of 3.5 and $12 \mathrm{~cm}$ and $67 \%$ decay for inner and outer radii of 7.5 and $24 \mathrm{~cm}$ for $10 \mathrm{~cm}$ distance from the surface of the coil and $98 \%$ decay for inner and outer radii of 0.5 and $3 \mathrm{~cm}, 97 \%$ decay for inner and outer radii of 1.5 and $6 \mathrm{~cm}, 94 \%$ decay for inner and outer radii of 3.5 and $12 \mathrm{~cm}$ and $90 \%$ decay for inner and outer radii of 7.5 and 24 $\mathrm{cm}$ for $20 \mathrm{~cm}$ distance from the surface of the coil. This analysis indicates that an increase in the circular coil's size decreases its decay rate with depth and increases the coil's efficiency for deep brain stimulation. It should be noted that after the distance of $20 \mathrm{~cm}$ from the surface of the coil, the normalized values of the induced electric field are closer to each other, showing the insignificant effect of changing the size of the coil on decay rate after $20 \mathrm{~cm}$ distance from the surface of the coil which agrees with all the data obtained so far.

\section{CONCLUSION}

TMS, as one of the techniques widely used for non-invasive treatment of psychiatric disorders, is developing and requires improvement. Since its performance is dependent on intensity and focality of the induced electric field, designing and utilizing coils that can fulfill both these requirements simultaneously is of high importance. In this study, effect of coil radius on decay rate and electric field penetration was analyzed. The results indicated that larger coils have less decay rate comparing to smaller coils while increasing the size of the coil over a certain threshold might not be optimal. The combination of all the mentioned factors can result in an optimal TMS coil which is the purpose of all the researchers in this area. 


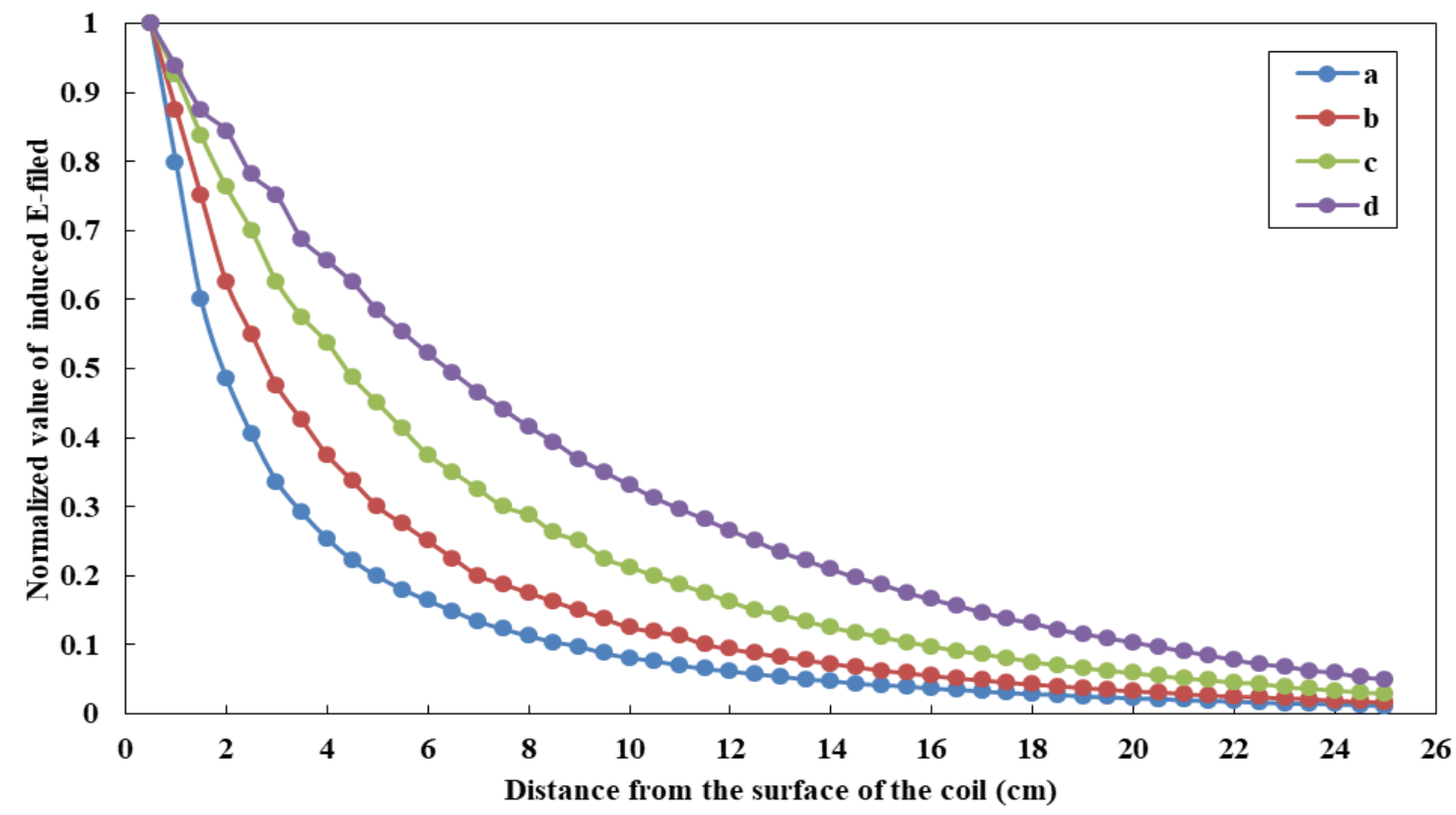

Figure 5: Normalized value of induced electric field for circular flat coils with varying inner and outer radii of a) $0.5 \mathrm{~cm}$ and $3 \mathrm{~cm}, \mathrm{~b}) 1.5 \mathrm{~cm}$ and $6 \mathrm{~cm}$, c) $3.5 \mathrm{~cm}$ and $12 \mathrm{~cm}$, d) $7.5 \mathrm{~cm}$ and $24 \mathrm{~cm}$, respectively.

\section{REFERENCES}

[1] Klomjai, W., Katz, R., Lackmy, A. "Basic principles of transcranial magnetic stimulation (TMS) and repetitive TMS (rTMS)," Annuals of physical and rehabilitation medicine, 58(4), 208-213 (2015).

[2] Merton, P. A. and Morton, H. B. "Stimulation of the cerebral cortex in the intact human subject," Nature, 285(5762), 227 (1980).

[3] Lefaucheur, J.P., André-Obadia, N., Antal, A., Ayache, S.S., Baeken, C., Benninger, D.H., Cantello, R.M., Cincotta, M., de Carvalho, M., De Ridder, D. and Devanne, H., "Evidence-based guidelines on the therapeutic use of repetitive transcranial magnetic stimulation (rTMS)," Clinical Neurophysiology, 125(11), 2150-2206 (2014).

[4] Ueno, S., Tashiro, T., and Harada, K., "Localized stimulation of neural tissues in the brain by means of a paired configuration of time-varying magnetic fields." Journal of Applied Physics64, no. 10: 5862-5864 (1988).

[5] Roth, Y., Zangen, A. and Hallett, M., "A coil design for transcranial magnetic stimulation of deep brain regions." Journal of Clinical Neurophysiology 19, no. 4: 361-370 (2002).

[6] Ishii, K., Matsuzaka, Y., Izumi, S., Abe, T., Nakazato, N., Okita, T., Yashima, Y., Takagi, T. and Nagatomi, R., "Evoked motor response following deep transcranial magnetic stimulation in a cynomolgus monkey." Brain Stimulation: Basic, Translational, and Clinical Research in Neuromodulation 1, no. 3: 300 (2008).

[7] Roth, Y., Amir, A., Levkovitz, Y. and Zangen, A., "Three-dimensional distribution of the electric field induced in the brain by transcranial magnetic stimulation using figure-8 and deep H-coils." Journal of Clinical Neurophysiology 24, no. 1: 31-38 (2007).

[8] Deng, Z.D., Lisanby, S.H. and Peterchev, A.V., "Electric field depth-focality tradeoff in transcranial magnetic stimulation: simulation comparison of 50 coil designs." Brain stimulation 6, no. 1: 1-13 (2013).

[9] Lu, M. and Ueno, S., "Comparison of the induced fields using different coil configurations during deep transcranial magnetic stimulation." PloS one 12, no. 6: e0178422 (2017).

[10] Meng, Q., Hong, E. and Choa, F.S., "Transcranial magnetic stimulation (TMS) coil designing for high electromagnetic field gradient generation." Brain Stimulation: Basic, Translational, and Clinical Research in Neuromodulation 10, no. 2 (2017): 499. 